\title{
Combined treatment with acetazolamide and cisplatin enhances chemosensitivity in laryngeal carcinoma Hep-2 cells
}

\author{
HONG GAO ${ }^{1}$, HAI DONG ${ }^{2}$, GUIJUN LI ${ }^{2}$ and HUI JIN ${ }^{1}$ \\ ${ }^{1}$ Department of Head and Neck Surgery, Tumor Hospital of Jilin Province, Changchun, Jilin 130012; ${ }^{2}$ Tonghua \\ Mining Group Limited Liability Company General Hospital, Baishan, Jilin 134300, P.R. China
}

Received April 20, 2017; Accepted January 17, 2018

DOI: $10.3892 /$ ol.2018.8529

\begin{abstract}
The aim of the present study was to determine whether acetazolamide (Ace) treatment enhances the chemosensitivity of Hep-2 laryngeal cells to cisplatin (Cis). At the logarithmic growth phase, Hep-2 cells were treated with Ace, Cis or both, and cell viability was detected using an MTT assay. The degree of apoptosis was detected using flow cytometry. Expression levels of apoptosis-related proteins, including BCL2 apoptosis regulator (bcl-2), BCL2 associated X (bax) and caspase-3, and of proliferation-related proteins, including proliferating cell nuclear antigen (PCNA) and tumor protein p53 (P53), were detected using western blotting. mRNA expression levels of aquaporin-1 (AQP1) in each group were detected using reverse transcription-polymerase chain reaction. Compared with the drugs used alone, treatment with both Ace and Cis displayed synergistic effects on the growth inhibition and apoptosis induction in Hep-2 cells. The Ace/Cis combination decreased the expression of PCNA but increased the expression of p53. In addition, the combination treatment decreased the ratio of bcl-2/bax and increased the expression of caspase-3, as well as decreased the expression of AQP1. These results demonstrated that the combined use of Ace and Cis enhanced the chemosensitivity of laryngeal carcinoma cells.
\end{abstract}

\section{Introduction}

Laryngeal cancer is the most common head and neck malignant tumor, and the second most common malignant neoplasm of the respiratory tract. The incidence of laryngeal cancer

Correspondence to: Dr Hui Jin, Department of Head and Neck Surgery, Tumor Hospital of Jilin Province, 1018 Huguang Road, Changchun, Jilin 130012, P.R. China

E-mail: jinhui3456789@163.com

Abbreviations: Ace, acetazolamide; Cis, cisplatin; AQP1, aquaporin-1; RT-PCR, reverse transcription-polymerase chain reaction; AceH, high concentration of acetazolamide; AceL, low concentration of acetazolamide

Key words: acetazolamide, cisplatin, Hep-2, apoptosis, proliferation, aquaporin-1, laryngeal cancer worldwide accounts for $\sim 1-5 \%$ of systemic tumors, and is also the second highest disease incidence after nasopharyngeal cancer in otolaryngology $(1,2)$. Laryngeal cancer seriously endangers human life and health. Currently, chemotherapy is one of the commonly used methods for treating laryngeal cancer, but side effects and drug resistance significantly hinder its effectiveness (3-5). The development of new anticancer agents and/or novel therapeutic strategies to enhance the chemosensitivity of laryngeal carcinoma cells is therefore the focus of extensive research.

Cisplatin (Cis) is one of the most effective and commonly used chemotherapeutic drugs for the treatment of locally advanced laryngeal carcinoma (6). Cis has been used as a single reagent or in combination with other agents to treat this disease (7). In addition, studies have reported that combined chemotherapy with Cis not only improves the prevention of resistance development in laryngeal cancer but also the survival rate of patients (8). However, Cis treatment alone may present several issues, such as easy tolerance or the use of higher doses after achieving appropriate efficacy and considerable toxic side effects, which further leads to poor treatment results (9). Thus the combined use of Cis with other cancer drugs, for appropriate compatibility or multi-target treatment, has been applied as a crucial therapeutic strategy for laryngeal cancer (10).

Acetazolamide (Ace) is a small heteroaromatic sulfonamide that binds to various carbonic anhydrases with high affinity, acting as a carbonic anhydrase (CA) inhibitor (11). Ace derivatives containing multiple charges do not efficiently cross the cell membrane and are restricted in binding to membrane-accessible carbonic anhydrases (for example, CAIX and potentially CAIV and CAXII) (12). Many sulfonamides inhibit the growth of tumor cells by inhibiting CAIX and CAXII. This finding is important for the treatment of tumors, and these two types of CA molecules have received much attention worldwide as novel potential anticancer targets. Ace has an antitumor metastasis effect, inducing the apoptosis of laryngeal cancer cells through the inhibition of AQP1 expression (13). Thus, the present study explored whether the Ace/Cis combination treatment could enhance the chemosensitivity of Hep-2 laryngeal cells.

In the current study, the drugs were used alone or in combination, and the results demonstrated that the Ace/Cis combination treatment was more effective in inhibiting proliferation of 
laryngeal carcinoma cells, enhancing cells apoptosis and decreasing the expression of AQP1.

\section{Materials and methods}

Cell culture and treatments. The laryngeal carcinoma cell line Hep-2 and human umbilical vein endothelial cells (HUVECs) were obtained from the American Type Culture Collection (Manassas, VA, USA). The Hep-2 cells were maintained in RPMI-1640 culture media (Gibco; Thermo Fisher Scientific, Inc., Waltham, MA, USA) supplemented with $10 \%$ fetal bovine serum (Gibco; Thermo Fisher Scientific, Inc.), $100 \mathrm{U} / \mathrm{ml}$ penicillin and streptomycin, and incubated in a $5 \% \mathrm{CO}_{2}$ humidified atmosphere at $37^{\circ} \mathrm{C}$. HUVECs were cultured in Dulbecco's modified Eagles Medium (Hyclone; GE Healthcare Life Sciences, Logan, UT, USA) with $5 \mathrm{mM}$ glucose and $10 \%$ fetal bovine serum in incubator containing $5 \% \mathrm{CO}_{2}$ at $37^{\circ} \mathrm{C}$. Experiments were performed at the logarithmic phase of growth of the cells.

For the drug treatments, Hep-2 cells were treated with Ace (a low concentration of $1 \times 10^{-8} \mathrm{~mol} / \mathrm{l}$, termed here AceL; or a high concentration of $5 \times 10^{-8} \mathrm{~mol} / 1$, termed here AceH), Cis $(1 \mu \mathrm{g} / \mathrm{ml})$ alone, or Cis in combination with Ace (AceL+Cis, or $\mathrm{AceH}+\mathrm{Cis}$ ) for $48 \mathrm{~h}$. Cells that were treated with equal volumes of vehicle were used as control. Ace was used at $1 \times 10^{-8}$ or $5 \times 10^{-8} \mathrm{~mol} / 1 \mathrm{l}$ all experiments. Cis was used at $1 \mu \mathrm{g} / \mathrm{ml}$ in all experiments. Both $\mathrm{Cis}$ and Ace were dissolved in dimethyl sulfoxide (DMSO) and then added to PBS to dilute to the final working concentrations. The final concentration of DMSO in cultures did not exceed $0.5 \%$. HUVECs were treated with AceH alone, Cis alone or in combination (AceH+Cis) or control (vehicle) for $48 \mathrm{~h}$.

Reverse transcription- polymerase chain reaction (RT-PCR), Total RNA from Hep-2 cells was extracted with TRIzol (Thermo Fisher Scientific, Inc.), according to the manufacturer's instructions. The mRNA expression of aquaporin-1 (AQP1) was detected by RT-PCR with GAPDH as an internal control. Reverse transcription was performed using PrimeScript RT kit with gDNA Eraser (Takara Biotechnology, Co., Ltd., Dalian, China). PCR analysis was performed using SYBR Green I (Takara Biotechnology Co., Ltd., Dalian, China). The primers used were as follows: Human AQP1, forward 5'-ACCCGC AACTTCTCAAAC-3' and reverse 5'-AGGCCAAGCCTC CTCTAT-3'; human GAPDH, forward 5'-ACCACAGTCCAT GCCATCAC-3' and reverse 5'-TCCACCACCCTGTTGCTG TA-3'. The PCR reaction was performed using the following conditions: $5 \mathrm{~min}$ pre-denaturation at $94^{\circ} \mathrm{C}$, followed by 35 cycles of $30 \mathrm{sec}$ denaturation at $94^{\circ} \mathrm{C}, 30 \mathrm{sec}$ annealing at $55^{\circ} \mathrm{C}$, and $30 \mathrm{sec}$ extension at $72^{\circ} \mathrm{C}$; finally, $10 \mathrm{~min}$ extension at $72^{\circ} \mathrm{C}$. The PCR products were detected using $1.5 \%$ agarose gel electrophoresis and the results were processed by gel imaging analysis system (SY-B175; Guangzhou Sunnymed Electronics Ltd., Guangzhou, China).

MTT assay. The cell viability of Hep-2 cells and HUVECs was measured by MTT assay (Sigma-Aldrich; Merck KGaA, Darmstadt, Germany). Hep-2 cells and HUVECs in logarithmic growth phase were plated in 96-well plates. Following $48 \mathrm{~h}$ of drug treatment as indicated, $200 \mu \mathrm{l}$ MTT $(5 \mathrm{mg} / \mathrm{ml})$ was added to each well. Cells were incubated with the MTT solution at $37^{\circ} \mathrm{C}$ for $4 \mathrm{~h}$. Then, $150 \mu \mathrm{l}$ DMSO was added for $5 \mathrm{~min}$. The optical density (OD) values were measured at $490 \mathrm{~nm}$ with a Versamax Microplate reader (Molecular Devices, LLC, Sunnyvale, CA, USA).

Annexin V apoptosis assay. Quantification of apoptotic cells was performed by Annexin V-fluorescein isothiocyanate (FITC)/propidium iodide (PI) double staining using a FITC-Annexin V Apoptosis Detection kit (BD Biosciences, San Jose, CA, USA). At the logarithmic growth phase, Hep-2 cells were placed in 6-well plates. The cells were treated with AceL, AceH, Cis, AceL+Cis, AceH+Cis, or vehicle for $48 \mathrm{~h}$. Then, cells were washed in PBS, digested with trypsin, and resuspended in calcium-enriched HEPES buffer. This suspension was stained with Annexin V-FITC and PI for $15 \mathrm{~min}$, as per the manufacturer's instructions. Finally, the cells were analyzed by FlowJo software (version 7.6.3; FlowJo, LLC, Ashland, OR, USA).

Western blot analysis. At the logarithmic growth phase, Hep-2 cells were seeded in 6-well plates. The cells were treated with AceL, AceH, Cis, AceL+Cis, AceH+Cis for 48 h. Cell protein was extracted by radioimmunoprecipitation assay lysis buffer (including protease inhibitor; Beijing ComWin Biotech Co., Ltd., Beijing, China), and the protein concentration was measured by coomassie brilliant blue staining. A total of $20 \mu \mathrm{g}$ protein per lane was resolved by SDS-PAGE ( $10 \%$ gel) and transferred to polyvinylidene fluoride membranes (Merck KGaA) for $2 \mathrm{~h}$. After washing, membranes were blocked in TBS/0.1\% Tween-20 (TBST) solution with 5\% non-fat dry milk for $1 \mathrm{~h}$. Primary antibodies against BCL2 associated X (bax; 1:1,000 dilution; ab32503, Abcam, Cambridge, UK), BCL2 apoptosis regulator (bcl-2; 1:1,000 dilution; ab32124, Abcam), caspase-3 (1:1,000 dilution; ab13585, Abcam), proliferating cell nuclear antigen (PCNA; 1:5,000 dilution; sc-400037, Santa Cruz Biotechnology, Inc., Dallas, TX, USA), tumor protein p53 (P53; 1:5000 dilution; sc-416469, Santa Cruz Biotechnology, Inc.) and $\beta$-actin (1:1,000 dilution; ab8226, Abcam) were diluted in TBST/3\% bovine serum albumin and incubated at room temperature for $1 \mathrm{~h}$. After washing, membranes were incubated at room temperature for $1 \mathrm{~h}$ with secondary antibody (goat anti-mouse, sc-2039; Santa Cruz Biotechnology, Inc.). Finally, membranes were developed with enhanced chemiluminescence substrate (PerkinElmer, Inc., Waltham, MA, USA) for 3-5 min. The results were analyzed with the Quantity One image analysis software (version 4.62; Bio-Rad Laboratories, Inc., Hercules, CA, USA).

Statistical analysis. The data were analyzed with SPSS version 19.0 (IBM SPSS Inc., Chicago, IL, USA). One-way analysis of variance was used to compare the differences between treatment groups. The Student-Newman-Keuls post hoc test was used to compare differences between two groups. All results were expressed as mean \pm standard deviation. $\mathrm{P}<0.05$ was considered to indicate a statistically significant difference.

\section{Results}

Combined Ace and Cis treatment effectively reduces viability in Hep-2 cells. Compared with the control group, the high 


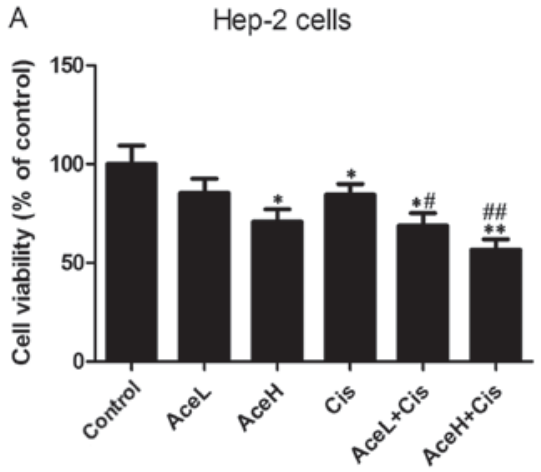

B Human umbilical vein endothelial cells

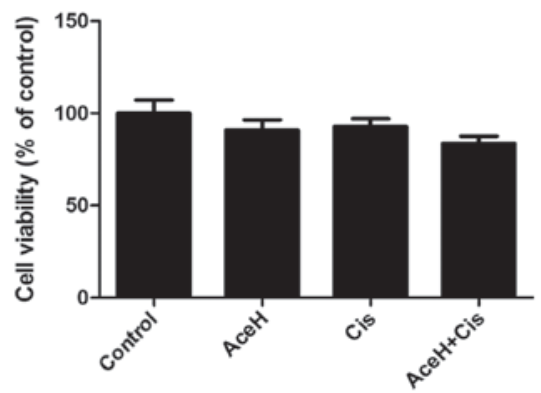

Figure 1. Effects of Ace and/or Cis treatment on cell viability. (A) Hep-2 cells were treated with AceL, AceH, Cis, AceL+Cis, AceH+Cis or vehicle for $48 \mathrm{~h}$. (B) Human umbilical vein endothelial cells were treated with AceH, Cis, AceH+Cis and vehicle for $48 \mathrm{~h}$. Cells viability was determined by MTT assay. Data were expressed as mean \pm standard deviation. ${ }^{*} \mathrm{P}<0.05$ and ${ }^{* *} \mathrm{P}<0.01$ compared with control group; ${ }^{\#} \mathrm{P}<0.05$ and ${ }^{\# \#} \mathrm{P}<0.01$ compared with Cis group. Ace, acetazolamide; Cis, cisplatin; L, low dose $1 \times 10^{-8} \mathrm{~mol} / \mathrm{l}$; $\mathrm{H}$, high dose $5 \times 10^{-8} \mathrm{~mol} / \mathrm{l}$.

Ace concentration (AceH, $\left.5 \times 10^{-8} \mathrm{~mol} / \mathrm{l}\right)$, Cis $(1 \mu \mathrm{g} / \mathrm{ml})$ and Cis combined with the low Ace concentration (AceL, $1 \times 10^{-8} \mathrm{~mol} /$ ) treatments significantly reduced viability of Hep-2 cells $(\mathrm{P}<0.05, \mathrm{P}<0.05$ and $\mathrm{P}<0.05$, respectively; Fig. $1 \mathrm{~A})$. The effects of the $\mathrm{AceH}$ and $\mathrm{Cis}$ combination treatment $(\mathrm{AceH}+\mathrm{Cis})$ were significantly different from the control group $(\mathrm{P}<0.01$; Fig. $1 \mathrm{~A})$. Notably, the AceL+Cis and AceH+Cis treatments were statistically different compared with the Cis alone group $(\mathrm{P}<0.05$ and $\mathrm{P}<0.01$, respectively; Fig. 1A). Compared with the control group, either AceH or Cis alone or their combination treatment had no effects on the viability of HUVECs (P>0.05; Fig. 1B). These results suggested that combined use of Ace and Cis reduced the viability of Hep-2 cells more effectively than $\mathrm{AceH}$ or $\mathrm{Cis}$ alone.

Combined Ace and Cis treatment effectively upregulates P53 expression in Hep-2 cells. P53 is a tumor suppressor gene that has an important role in the negative regulation of cell proliferation. Treatment with the Ace/Cis combination significantly increased the expression levels of P53 (Fig. 2A), as both $\mathrm{AceL}+\mathrm{Cis}$ and $\mathrm{AceH}+\mathrm{Cis}$ treatments resulted in significantly increased $\mathrm{P} 53$ protein expression levels compared with the control group ( $\mathrm{P}<0.01$ for both; Fig. $2 \mathrm{~B})$. In addition, AceH+Cis more effectively increased the expression of P53 compared with either the AceH or Cis alone treatments $(\mathrm{P}<0.05$; Fig. 2B). These results suggested that the effects of Ace were dose-dependent, and that the combined use of Ace and Cis inhibited the proliferation of Hep-2 cells more effectively than $\mathrm{AceH}$ or Cis alone.
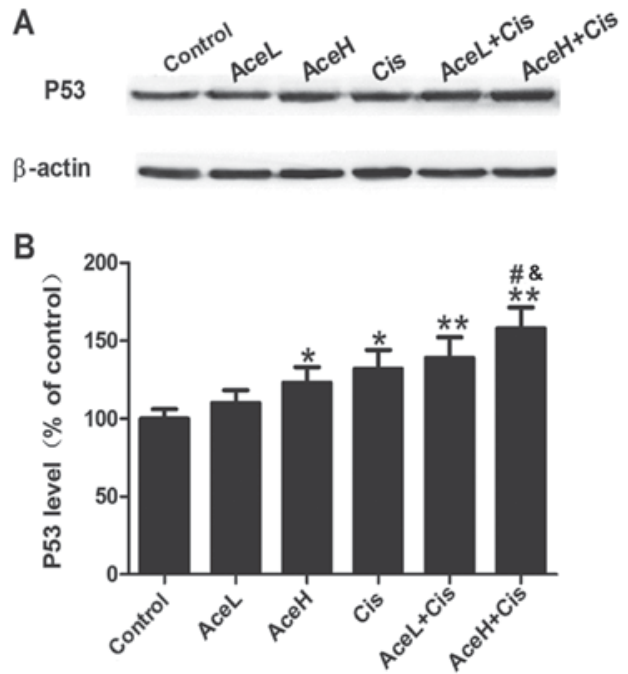

Figure 2. Effects of Ace and/or Cis treatment on P53 expression in Hep-2 cells. (A) Representative images from western blot analysis of the protein expression levels of P53 in Hep- 2 cells. $\beta$-actin was used as a housekeeping control. (B) Quantification of P53 protein expression levels following different drug treatments for $48 \mathrm{~h}$. Data were expressed as mean \pm standard deviation. ${ }^{*} \mathrm{P}<0.05$ and ${ }^{* *} \mathrm{P}<0.01$ compared with control group; ${ }^{*} \mathrm{P}<0.05$ compared with Cis group; ${ }^{\circledR} \mathrm{P}<0.05$ compared with $\mathrm{AceH}$ group. Ace, acetazolamide; $\mathrm{Cis}$, cisplatin; P53, tumor protein $\mathrm{p} 53$; L, low dose $1 \times 10^{-8} \mathrm{~mol} / \mathrm{l} ; \mathrm{H}$, high dose $5 \times 10^{-8} \mathrm{~mol} / 1$.
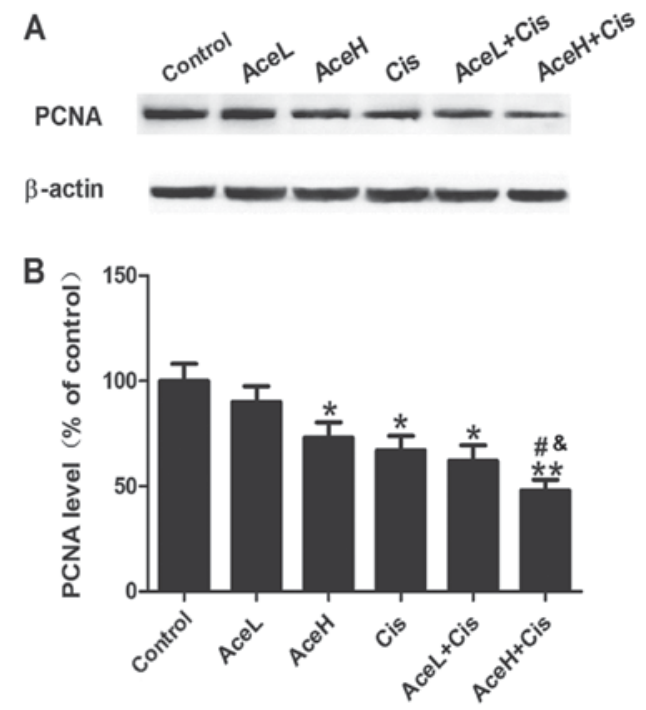

Figure 3. Effects of Ace and/or Cis treatment on PCNA expression in Hep-2 cells. (A) PCNA expression in Hep-2 cells was determined by western blot analysis. Protein loading was assessed by probing for $\beta$-actin. Experiments were duplicated in independent cultures. Representative blot images are shown. (B) Quantification of PCNA protein expression levels following drug treatments for $48 \mathrm{~h}$. Data expressed as mean \pm standard deviation. ${ }^{*} \mathrm{P}<0.05$ and ${ }^{* *} \mathrm{P}<0.01$ compared with control group; ${ }^{\#} \mathrm{P}<0.05$ compared with Cis group; ${ }^{\circledR} \mathrm{P}<0.05$ compared with AceH group. Ace, acetazolamide; Cis, cisplatin; PCNA, proliferating cell nuclear antigen; L, low dose $1 \times 10^{-8} \mathrm{~mol} / 1$; $\mathrm{H}$, high dose $5 \times 10^{-8} \mathrm{~mol} / \mathrm{l}$.

Combined Ace and Cis treatment effectively inhibits proliferation in Hep-2 cells. Next, to observe the effects of different drug treatments on cell proliferation, PCNA protein expression was examined, as an indicator of tumor cell proliferation activity (Fig. 3A). The results demonstrated that AceH, Cis and Ace L+Cis treatments significantly inhibited the expression 


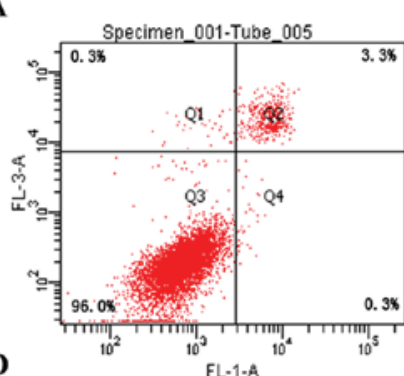

B

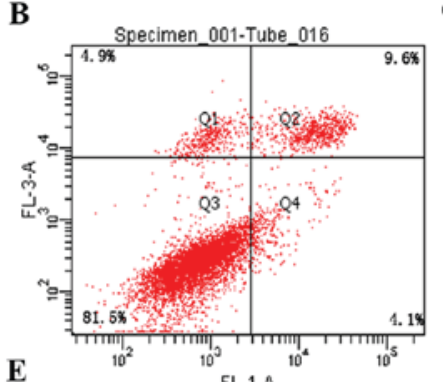

C

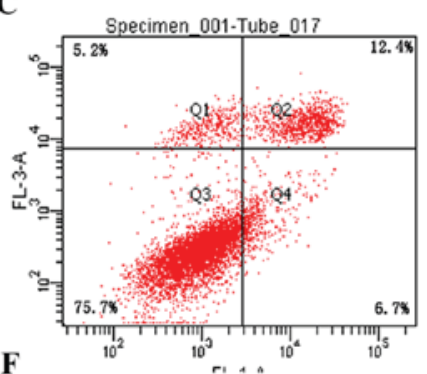

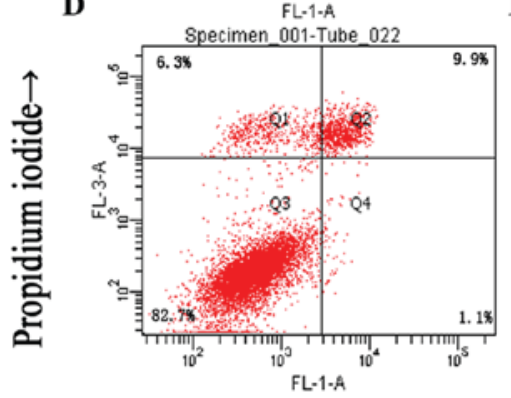
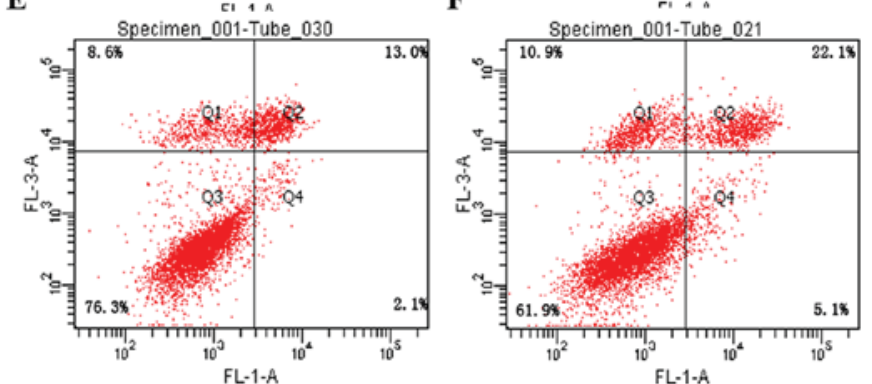

Annexin V-FITC $\rightarrow$
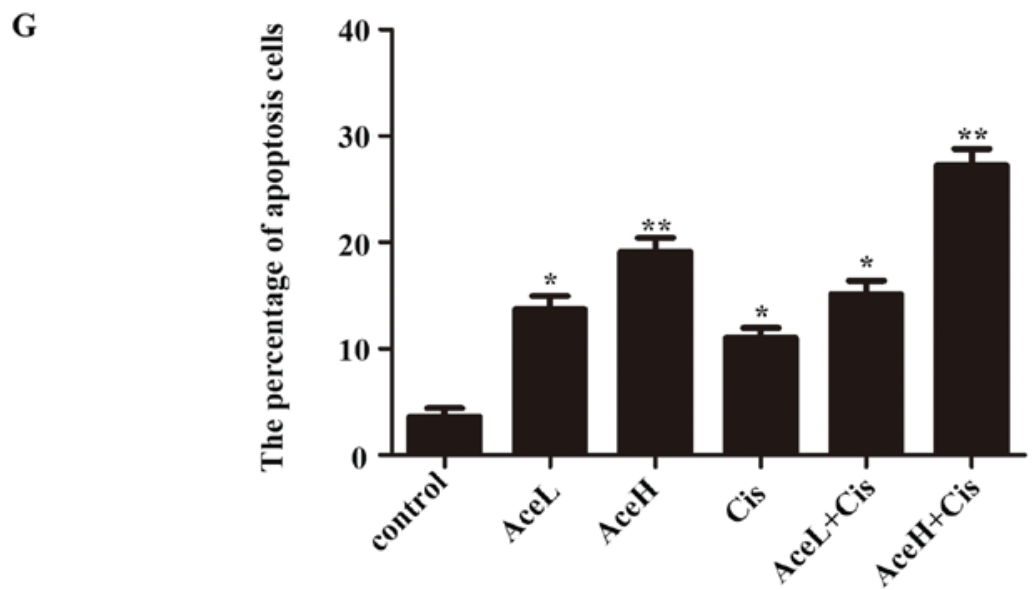

Figure 4. Combined effect of Ace and Cis on cell apoptosis of Hep-2 cells. Flow cytometric analysis of Annexin V/propidium iodide double staining in Hep-2 cells following drug treatments for 48 h. (A) Untreated (control), (B) AceL, (C) AceH, (D) Cis, (E) AceL+Cis, (F) AceH+Cis, and (G) quantification of A-F. ${ }^{*} \mathrm{P}<0.05$ and ${ }^{* *} \mathrm{P}<0.01$ compared with control group. Ace, acetazolamide; Cis, cisplatin; L, low dose $1 \times 10^{-8} \mathrm{~mol} / 1 ; \mathrm{H}$, high dose $5 \times 10^{-8} \mathrm{~mol} / 1$.

of PCNA compared with the control group $(\mathrm{P}<0.05$ for all; Fig. 3B), and that the effect of $\mathrm{AceH}+\mathrm{Cis}$ treatment was more significant compared with the control group ( $\mathrm{P}<0.01$; Fig. $3 \mathrm{~B})$. In addition, PCNA expression levels following AceH+Cis treatment were significantly decreased compared with the AceH or $\mathrm{Cis}$ alone treatments $(\mathrm{P}<0.05$ for both; Fig. 3B). These results suggested that the effects of Ace on cell proliferation were dose-dependent, and that combined treatment of Ace and Cis decreased the proliferation of Hep- 2 cells more effectively than AceH or Cis alone.

Combined Ace and Cis treatment effectively promotes apoptosis in Hep-2 cells. To verify whether combination therapy is superior to the use of each drug alone, the apoptosis rate of Hep-2 cells was further evaluated using flow cytometric analysis. After $48 \mathrm{~h}$, the combined treatment AceH+Cis significantly induced the apoptosis of Hep-2 cells $(\mathrm{P}<0.01)$. In addition, the effects of AceH+Cis combination treatment on the apoptosis of Hep-2 cells were more pronounced compared with the AceH or AceL+Cis treatments ( $\mathrm{P}<0.05$; Fig. 4).

Bcl-2, bax and caspase-3 are apoptosis-related proteins. To further assess the apoptotic response of Hep- 2 cells following the treatments, the expression levels of these proteins were analyzed by western blotting (Fig. 5A). The Ace/Cis combination treatment significantly reduced the bcl-2/bax expression ratio $(\mathrm{P}<0.05$; Fig. $5 \mathrm{~B})$, and increased the expression of caspase-3 protein $(\mathrm{P}<0.01$; Fig. $5 \mathrm{C})$, compared with the control group. AceL, AceH, Cis and AceL+Cis treatments significantly reduced the bcl-2/bax ratio compared with the control group $(\mathrm{P}<0.05$ for all; Fig. 5B). However, compared with the control group, the $\mathrm{AceH}+\mathrm{Cis}$ treatment displayed a more significant reduction of the bcl-2/bax ratio compared with the control $(\mathrm{P}<0.01$; Fig. 5B). The AceL+Cis treatment significantly reduced the bcl-2/bax ratio compared with the Cis group $(\mathrm{P}<0.05$; Fig. 5B). The effect of $\mathrm{AceH}+\mathrm{C}$ is was more significant compared with either AceH or Cis alone on the inhibition of bcl-2/bax $(\mathrm{P}<0.05$ and $\mathrm{P}<0.01$, respectively; Fig. 5B). 
A

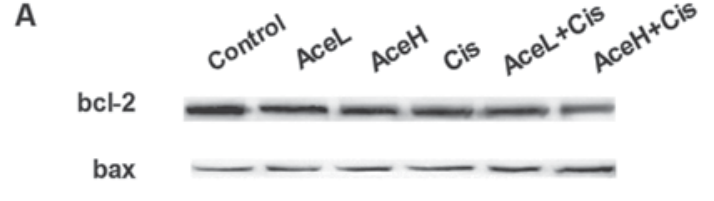

$\begin{gathered}\text { Caspase-3 } \\ \beta \text {-actin }\end{gathered}--$

B

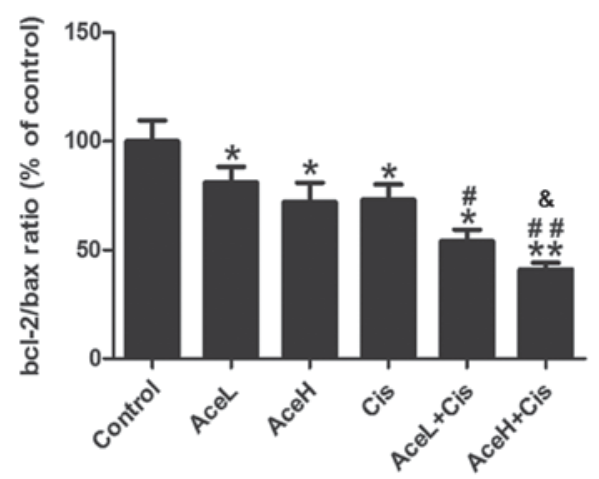

C

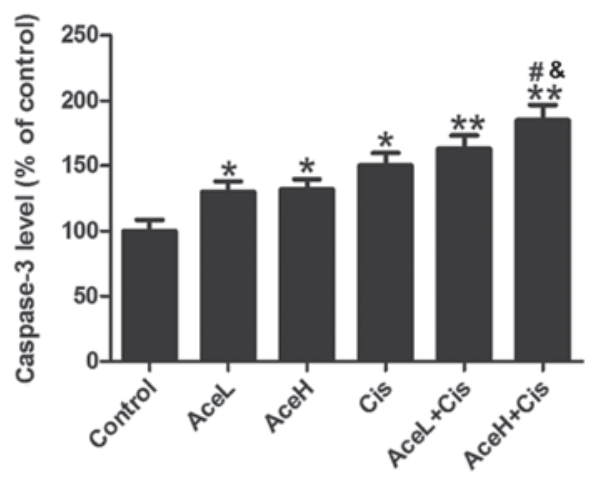

Figure 5. Effects of Ace and/or Cis treatment on the expression of apoptosis-related proteins. (A) Bcl-2, bax and caspase-3 expression in Hep-2 cells was determined by western blot analysis. Protein loading was assessed by probing for $\beta$-actin. (B) Quantification of the bcl-2/bax protein expression ratio following drug treatments for $48 \mathrm{~h}$. (C) Quantification of caspase-3 protein expression levels following drug treatments for $48 \mathrm{~h}$. Data expressed as mean \pm standard deviation. " $\mathrm{P}<0.05$ and ${ }^{* *} \mathrm{P}<0.01$ compared with control group; ${ }^{\#} \mathrm{P}<0.05$ and ${ }^{\# \#} \mathrm{P}<0.01$ compared with $\mathrm{Cis}$ group; ${ }^{\circledR} \mathrm{P}<0.05$ compared with AceH group. Ace, acetazolamide; Cis, cisplatin; Bcl-2, BCL2 apoptosis regulator; Bax, BCL2 associated X; L, low dose $1 \times 10^{-8} \mathrm{~mol} / \mathrm{l} ; \mathrm{H}$, high dose $5 \times 10^{-8} \mathrm{~mol} / 1$.

As far as the caspase-3 levels are concerned, AceL, AceH and $\mathrm{Cis}$ treatments alone significantly increased caspase-3 expression compared with the control group $(\mathrm{P}<0.05$ for all; Fig. 5C). The expression of caspase- 3 protein was significantly increased in the Ace $\mathrm{L}+\mathrm{Cis}$ and $\mathrm{AceH}+\mathrm{Cis}$ treatment groups compared with the control group $(\mathrm{P}<0.01$ for both; Fig. 5C). Furthermore, the effects of AceH+Cis treatment on increasing caspase-3 were more significant compared with $\mathrm{AceH}$ or $\mathrm{Cis}$ alone $(\mathrm{P}<0.05$ for both; Fig. 5C).

These results suggested that the effects of Ace on cell apoptosis were dose-dependent, and that combined treatment of Ace and Cis promoted apoptosis in Hep-2 cells more effectively than AceH or Cis alone.

Combined Ace and Cis treatment effectively decreased the expression of AQP1 mRNA. Combined treatment with Ace/Cis
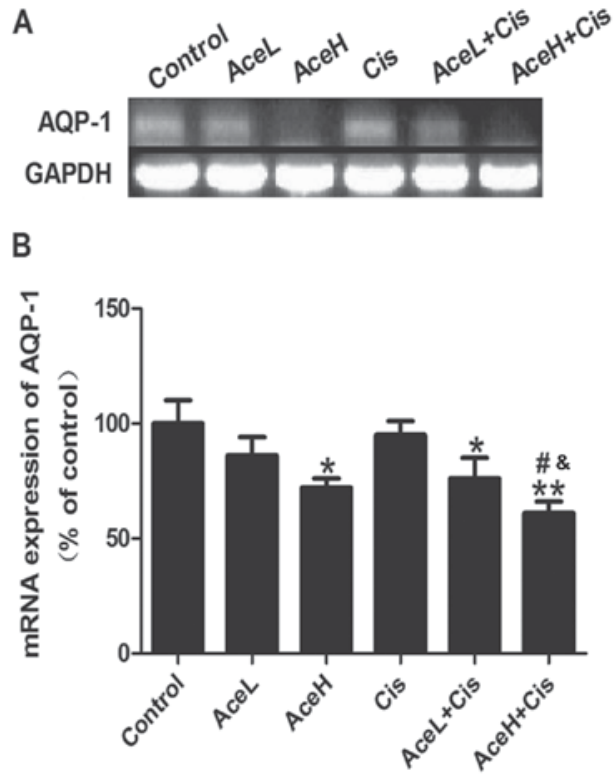

Figure 6. Effects of Ace and/or Cis treatment on the mRNA expression of AQP1 in Hep-2 cells. (A) Representative electrophoretic patterns of PCR products following the indicated drug treatments. GAPDH was used as the loading control. (B) Quantification of AQP1 mRNA expression levels following the indicated drug treatments. Data expressed as mean \pm standard deviation. ${ }^{*} \mathrm{P}<0.05$ and ${ }^{* *} \mathrm{P}<0.01$ compared with control group; ${ }^{\#} \mathrm{P}<0.05$ compared with Cis group; ${ }^{\&} \mathrm{P}<0.05$ compared with $\mathrm{AceH}$ group. Ace, acetazolamide; Cis, cisplatin; AQP1, aquaporin-1; L, low dose $1 \times 10^{-8} \mathrm{~mol} / \mathrm{l}$; $\mathrm{H}$, high dose $5 \times 10^{-8} \mathrm{~mol} / \mathrm{l}$.

markedly decreased the expression of AQP1 mRNA in Hep-2 cells (Fig. 6A). Both AceH and AceL+Cis treatments decreased the expression of AQP1 mRNA in Hep-2 cells compared with the control group $(\mathrm{P}<0.05$ for both; Fig. 6B). AceH+Cis treatment also decreased the expression of AQP1 mRNA in Hep-2 cells compared with the control group $(\mathrm{P}<0.01$; Fig. 6B). Notably, the effects of $\mathrm{AceH}+\mathrm{Cis}$ treatment on the expression of AQP1 mRNA were more significant than those of AceH or Cis alone $(\mathrm{P}<0.05$ for both; Fig. 6B). These results suggested that combined treatment of Ace and Cis decreased the expression of AQP1 mRNA more effectively than AceH or Cis alone.

\section{Discussion}

In the present study, the results demonstrated that combined treatment with Ace and Cis inhibited the proliferation of Hep-2 cells more effectively than each drug alone. Treatment of laryngeal cancer cells with the Ace/Cis combination enhanced the expression of apoptosis-related proteins and decreased the expression of the proliferation marker PCNA. Finally, the combination treatment increased the expression of the tumor suppressor protein $\mathrm{p} 53$, thereby affecting the expression of AQP1.

Laryngeal cancer is the most common head and neck malignant tumor. Currently, $\mathrm{Cis}$ is one of the commonly used drugs for laryngeal cancer treatment (14). However, Cis alone has many shortcomings, such as drug resistance, nephrotoxicity, gastrointestinal toxicity, bone marrow suppression and ototoxicity and other considerable toxic side effects, which limit the use of this drug (15). Thus, currently the chemotherapy regimens for laryngeal cancer are based on Cis combination 
therapies. Studies have reported that resveratrol significantly increases the sensitivity of Hep-2 laryngeal cancer cell lines to $\mathrm{C}$ is (16). Ginsenoside $\mathrm{Rh} 2$ or Cis alone can induce the apoptosis of laryngeal carcinoma cells, and the combination of the two drugs displays a synergistic effect, inducing a more obvious anticancer effect (10). In addition, several studies have demonstrated that other compounds bound to Cis constitute an effective method to overcome drug resistance and reduce adverse side effects $(17,18)$. Ace has received much attention in the field of cerebrovascular disease and cancer (19). Previous studies have indicated that a small molecule-drug conjugated product, which was obtained using Ace and monomethylated toast oil, exerted an effective antitumor activity in mice with renal cell carcinoma (12). Additional studies have demonstrated that Ace, as a treatment in mice with transplanted human colon tumors, significantly suppressed tumor growth, with tumor inhibition rates as high as $88.28 \%$ (20,21). Studies have indicated that the commonly used doses of Cis in antitumor experiments in vitro are $0.25-20 \mu \mathrm{g} / \mathrm{ml}(6-25)$. To observe the effect of combination therapy and minimize the generation of side effects, the present study selected a relatively low effective dose of $1 \mu \mathrm{g} / \mathrm{ml}$ Cis. Notably, $1 \mu \mathrm{g} / \mathrm{ml} \mathrm{Cis}$ effectively inhibits the proliferation of laryngeal cancer cells, the effect of which was less than what has been previously observed with $5 \mu \mathrm{g} / \mathrm{ml}$ Cis $(22,25,26)$. In the present study, the anticancer effect of the combination therapy was significantly improved compared with that observed with $1 \mu \mathrm{g} / \mathrm{ml} \mathrm{Cis} \mathrm{alone}$ treatment, and reached or exceeded the effect of $5 \mu \mathrm{g} / \mathrm{ml} \mathrm{Cis,}$ suggesting that the combination therapy could improve the chemical sensitivity of laryngeal cancer cells. Furthermore, the combination of Ace and Cis displayed little cytotoxicity on normal HUVECs. Taken together, the combined use of these drugs not only reduced the toxicity of Cis but also promoted the chemotherapy sensitivity of laryngeal cancer to $\mathrm{Cis}$, suggesting that the Ace/Cis combination treatment may potentially be a useful therapeutic option for patients diagnosed with laryngeal cancer.

To further characterize the potential mechanism underlying the synergistic effects of the combined Ace/Cis treatment, cell apoptosis experiments were performed. The results demonstrated that compared to treatment with Ace or Cis alone, the cell apoptosis induced by their combination was significantly increased, indicating that Ace enhances Cis-induced cell apoptosis on Hep-2 cells. The main mechanism underlying the antitumor effect of Cis involves the induction of tumor cell apoptosis. Studies have reported that changes in tumor cell apoptosis primarily reflect abnormal cell apoptotic signal transduction pathways and the abnormal expression of apoptosis-related factors (22).

Ace has broad spectrum inhibition effects on carbonic anhydrase in cells from different tissues, including human erythrocytes, pancreas, and the central nervous system (12). Studies have previously reported that Ace had an important role in inhibiting angiogenesis, and that this function may be associated with the upregulation of DNA-related proteins as observed by serum proteomics (19). To obtain a better understanding of the mechanisms by which the Ace/Cis combination enhances the sensitivity of laryngeal carcinoma Hep-2 cells to Cis, the present study further investigated the expression levels of key proteins that regulate the proliferation and apoptosis of laryngeal cancer cells. The present results demonstrated that treatment with the Ace/Cis combination significantly reduced the bcl-2/bax ratio and increased the expression of caspase-3 protein. Regulation of cell apoptosis protein is generally performed by both apoptosis-inhibiting and apoptosis-inducing proteins. Among these proteins, the BCL2 family is an important apoptosis regulator; bcl-2 is an apoptosis-inhibiting protein, while bax is an apoptosis-inducing protein. To achieve anti-apoptosis effects, Bcl-2 and other apoptosis inhibitors block caspase- 3 activity and degrade the caspase- 3 substrate poly(ADP-ribose) polymerase (27).

Combined treatment with Ace and Cis also more effectively inhibited the proliferation of Hep-2 cells than each drug used alone. PCNA, a well-established proliferation marker, is an acidic polypeptide synthesized and expressed in proliferating laryngeal cancer cells and an essential factor in cell synthesis (28). PCNA is expressed in the nucleus during the late G1 phase, increases during the $\mathrm{S}$ phase, and declines during the G2 and M phases. Thus, the expression levels of PCNA have a clear correlation with cell proliferation, and are used as an indicator of cell proliferation $(29,30)$. The expression levels of PCNA in cells treated with a high concentration of Ace combined with $\mathrm{Cis}$ were decreased compared with either single treatment group, suggesting that the Ace and Cis combination inhibited the expression of PCNA, consistent with previous studies (28-30).

The tumor suppressor gene p53 is one of the most well-studied tumor suppressor genes in the last decade (31). P53 serves a role in cancer suppression in a variety of mechanisms, referred to as the 'molecular police' to maintain the stability of the human genome. By contrast, the mutant p53 gene has a role as a proto-oncogene, which promotes the development and progression of tumors. P53 gene mutations are observed in almost all human tumors (32). In tumor cells, mutant p53 loses its ability to monitor cells, leading to continuous proliferation and lack of cell apoptosis. The mismatched DNA cells can still enter the $\mathrm{S}$ phase, eventually leading to the occurrence of cancer (33). In the present study, treatment of Hep-2 cells with the combination of drugs reduced the expression of p53, which may explain the observed inhibition of proliferation.

In the present study, the results also revealed that the Ace/Cis combination therapy reduced the expression of AQP1 more effectively than either agent alone. Studies have reported that Ace is one of the most widely used aquaporin inhibitors, and its site of action is consistent with the distribution of AQP1. The Ace-mediated inhibition of tumor metastasis may be associated with the downregulation of AQP1 protein expression (34). AQP1 is expressed in throat vascular endothelial cells, and this protein can quickly transport water into the surrounding tissue fluid through capillary endothelial cells, which is consistent with the needs of throat cell metabolism. Therefore, AQP1 has an important role in maintaining the normal physiological function of the throat (35). It has been confirmed that AQP1 expression in laryngeal carcinoma vascular endothelial cells is significantly higher than that in adjacent normal tissues (36). This finding confirms that AQP1 can increase tumor vascular permeability and promote the rapid water transport in tumor cells, thereby promoting tumor angiogenesis (36). The increased expression of AQP1 alters the osmotic pressure of tumor cell membrane, 
changes the tumor cell volume and shape, and subsequently affects the surrounding matrix infiltration, promoting tumor cell proliferation. By contrast, if the expression of AQP1 is reduced, then the rate of tumor migration decreases (37).

In summary, the present results have demonstrated for the first time that Ace/Cis combination treatment inhibited cell proliferation and promoted apoptosis in Hep-2 cells, while decreasing the expression of AQP1. These findings suggest a potential clinical application of the combination regimen for the treatment of laryngeal cancer. However, a limitation of the current report is the lack of validation using in vivo experiments. Further studies will be required in the future to validate these results in an in vivo setting.

\section{Funding}

No funding was received.

\section{Availability of data and materials}

All data generated or analyzed during this study are included in this published article.

\section{Authors' contributions}

HG made substantial contributions to the conception and design of the study. HD and GL provided materials for the study. HD, GL and HJ contributed to the collection and assembly of data. GL and HJ analyzed and interpreted the data. All authors made contributions in writing the manuscript. All authors approved the final manuscript.

\section{Ethics approval and consent to participate}

Not applicable.

\section{Consent for publication}

Not applicable.

\section{Competing interests}

The authors declare that they have no competing interests.

\section{References}

1. Markowski J, Sieroń AL, Kasperczyk K, Ciupińskakajor M, Auguściakduma A and Likus W: Expression of the tumor suppressor gene hypermethylated in cancer 1 in laryngeal carcinoma. Oncol Lett 9: 2299-2302, 2015.

2. Pei SG, Wang JX, Wang XL, Zhang QJ and Zhang H: Correlation of survivin, p53 and Ki-67 in laryngeal cancer Hep-2 cell proliferation and invasion. Asian Pac J Trop Med 8: 636-642, 2015.

3. Yang X, An L and Li X: Arsenic trioxide induced endoplasmic reticulum stress in laryngeal squamous cell line Hep-2 cells. Auris Nasus Larynx 41: 81-83, 2014.

4. Cheng JZ, Yu D, Zhang H, Jin CS, Liu Y, Zhao X, Qi XM and Liu XB: Inhibitive effect of IL-24 gene on CD133(+) laryngeal cancer cells. Asian Pac J Trop Med 7: 867-872, 2014.

5. Min JW, Kim KI, Kim HA, Kim EK, Noh WC, Jeon HB, Cho DH, Oh JS, Park IC, Hwang SG and Kim JS: INPP4B-mediated tumor resistance is associated with modulation of glucose metabolism via hexokinase 2 regulation in laryngeal cancer cells. Biochem Biophys Res Commun 440: 137-142, 2013.
6. Kang R, Wang ZH, Wang BQ, Zhang CM, Gao W, Feng Y, Bai T, Zhang HL, Huang-Pu H and Wen SX: Inhibition of autophagy-potentiated chemosensitivity to cisplatin in laryngeal cancer Hep-2 cells. Am J Otolaryngol 33: 678-684, 2012.

7. Wang $\mathrm{D}$ and $\mathrm{Wu} \mathrm{X}$ : In vitro and in vivo targeting of bladder carcinoma with metformin in combination with cisplatin. Oncol Lett 10: 975-981, 2015.

8. Cimbora-Zovko T, Fritz G, Mikac N and Osmak M: Downregulation of RhoB GTPase confers resistance to cisplatin in human laryngeal carcinoma cells. Cancer Lett 295: 182-190, 2010.

9. Liu T, Peng H, Zhang M, Deng Y and Wu Z: Cucurbitacin B, a small molecule inhibitor of the Stat 3 signaling pathway, enhances the chemosensitivity of laryngeal squamous cell carcinoma cells to cisplatin. Eur J Pharmacol 641: 15-22, 2010.

10. Hong-Jun XU, Meng Y and Sun YX: Effects of ginsenoside Rh_2 associated with cisplatin on human laryngeal squamous cell carcinoma strain Hep-2. Chin J Lab Diagn 10: 506-508, 2005.

11. Ahlskog JK, Dumelin CE, Trüssel S, Mårlind J and Neri D: In vivo targeting of tumor-associated carbonic anhydrases using acetazolamide derivatives. Bioorg Med Chem Lett 19: 4851-4856, 2009.

12. Cazzamalli S, Corso AD and Neri D: Linker stability influences the anti-tumor activity of acetazolamide-drug conjugates for the therapy of renal cell carcinoma. J Control Release 246: 39-45, 2017.

13. Guan G and Dong Z: Effect of inhibiting aquaporin-1 on proliferation and apoptosis of the Hep-2 cell. Lin Chuang Er Bi Yan Hou Ke Za Zhi 20: 988-991, 2006 (In Chinese).

14. Zhang X, Zhang L and Zou Y: Research evolution of cisplatin antitumor drugs and cisplatin-loaded. China Mod Med 18: 25-27, 2011.

15. Dasari S and Tchounwou PB: Cisplatin in cancer therapy: Molecular mechanisms of action. Eur J Pharmacol 740: 364-378, 2014.

16. Zhan S, Ping W, Yin W and Wei Z: Enhancement effect of resveratrol on sensitivity of laryngeal carcinoma Hep-2 cells to cisplatin and its mechanism. J Jilin Uni 41: 282-286, 2015.

17. Cui Y, Chao W, Xu D, Meng W and Quan X: AstragalosideII inhibits autophagic flux and enhance chemosensitivity of cisplatin in human cancer cells. Biomed Pharmacother 81: 166-175, 2016.

18. Singh M, Bhui K, Singh R and Shukla Y: Tea polyphenols enhance cisplatin chemosensitivity in cervical cancer cells via induction of apoptosis. Life Sci 93: 7-16, 2013.

19. Xiao ZH, Lin W and Hai W: Recent progress in clinical application of the carbonic anhydrase inhibitor, acetazolamide. Chin J New Drugs 17: 1390-1394, 2008.

20. Kong B, Xiao-Hua WU and Yong LI: Effects of aquaporin protein inhibitor acetazolamide on xenograft tumor growth of colon cancer in nude mice. China J Modern Med 20: 1466-1465, 2010.

21. Bin K and Shi-Peng Z: Acetazolamide inhibits aquaporin-1 expression and colon cancer xenograft tumor growth. Hepatogastroenterology 58: 1502-1506, 2011.

22. Nör C, Zhang Z, Warner KA, Bernardi L, Visioli F, Helman JI, Roesler R and Nör JE: Cisplatin induces Bmi-1 and enhances the stem cell fraction in head and neck Cancer. Neoplasia 16: 137-146, 2014.

23. Xu YY, Wu TT, Zhou SH, Bao YY, Wang QY, Fan J and Huang YP: Apigenin suppresses GLUT-1 and p-AKT expression to enhance the chemosensitivity to cisplatin of laryngeal carcinoma Hep-2 cells: An in vitro study. Int J Clin Exp Pathol 7: 3938-3947, 2014.

24. Ju SM, Kang JG, Bae JS, Pae HO, Lyu YS and Jeon BH: The flavonoid apigenin ameliorates cisplatin-induced nephrotoxicity through reduction of p53 activation and promotion of PI3K/Akt pathway in human renal proximal tubular epithelial cells. Evid Based Complement Alternat Med 2015: 186436, 2015.

25. Gao W, Ying L, Qin R, Liu D and Feng Q: Silence of fibronectin 1 increases cisplatin sensitivity of non-small cell lung cancer cell line. Biochem Biophys Res Commun 476: 35-41, 2016.

26. Yamauchi K, Sakurai H, Kimura T, Wiriyasermkul P, Nagamori S, Kanai Y and Kohno N: System L amino acid transporter inhibitor enhances anti-tumor activity of cisplatin in a head and neck squamous cell carcinoma cell line. Cancer Lett 276: 95-101, 2009.

27. Yang XK, Zheng F, Chen JH, Gao QL, Lu YP, Wang SX, Wang CY and Ma D: Relationship between expression of apoptosis-associated proteins and caspase-3 activity in cisplatin-resistant human ovarian cancer cell line. Ai Zheng 21: 1288-1291, 2002 (In Chinese). 
28. Shi-Hong MA and Tan WH: Expression and clinical significance of FHIT and PCAN protein in endometrial carcinoma. J Harbin Med Uni 43: 62-65, 2009.

29. Sittel C, Ruiz S, Volling P, Kvasnicka HM, Jungehülsing M and Eckel HE: Prognostic significance of Ki-67 (MIB1), PCNA and p53 in cancer of the oropharynx and oral cavity. Oral Oncol 35: 583-589, 1999.

30. Luo GQ, Dai D and Dong-Mei WU: The clinical implication of experession for both PCNA and p53 protein in larynx cancer patients. Ningxia Med J, 2001.

31. Wang ZM, Chang-Shao XU and Sun YM: The clinic value of the expression of $\mathrm{p} 53$, p16, PCNA protein in esophageal carcinoma. Bull Chin Cancer 15:61-62, 2006.

32. Zeng R: Expression of p53, p21, PCNA and COX-2 and its relationship with recurrence in the early-stage laryngeal cancer with negative surgical margin. Lin Chung Er Bi Yan Hou Tou Jing Wai Ke Za Zhi 30: 349-352, 2016 (In Chinese).
33. Pfister NT, Yoh KE and Prives C: p53, DNA damage, and NAD+ homeostasis. Cell Cycle 13: 1661-1662, 2014.

34. Hayashi Y, Edwards NA, Proescholdt MA, Oldfield EH and Merrill MJ: Regulation and function of aquaporin-1 in glioma cells. Neoplasia 9: 777-787, 2007.

35. Guan B, Sun L and Dong Z: The expression and distribution of Aquaporin 1 and Aquaporin 4 in laryngeal carcinoma and its significance. Chin J Clin Oncol 21: 269-272, 2005.

36. Musumeci G, Leonardi R, Carnazza ML, Cardile V, Pichler K, Weinberg AM and Loreto C: Aquaporin 1 (AQP1) expression in experimentally induced osteoarthritic knee menisci: An in vivo and in vitro study. Tissue Cell 45: 145-152, 2013.

37. Hoque MO, Soria JC, Woo J, Lee T, Lee J, Jang SJ, Upadhyay S, Trink B, Monitto C, Desmaze C, et al: Aquaporin 1 is overexpressed in lung cancer and stimulates NIH-3T3 cell proliferation and anchorage-independent growth. Am J Pathol 168: 1345-1353, 2006. 